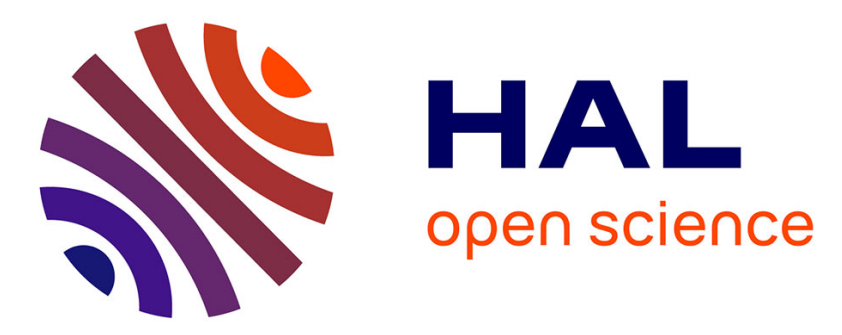

\title{
Flexible hydrofoil optimization for the 35th America's Cup with constrained EGO method
}

Matthieu Sacher, Mathieu Durand, Elisa Berrini, Frédéric Hauville, Régis

Duvigneau, Olivier Le Maitre, Jacques-André Astolfi

\section{- To cite this version:}

Matthieu Sacher, Mathieu Durand, Elisa Berrini, Frédéric Hauville, Régis Duvigneau, et al.. Flexible hydrofoil optimization for the 35th America's Cup with constrained EGO method. Ocean Engineering, 2018, 157, pp.62 - 72. 10.1016/j.oceaneng.2018.03.047 . hal-01785595

\section{HAL Id: hal-01785595 \\ https://inria.hal.science/hal-01785595}

Submitted on 4 May 2018

HAL is a multi-disciplinary open access archive for the deposit and dissemination of scientific research documents, whether they are published or not. The documents may come from teaching and research institutions in France or abroad, or from public or private research centers.
L'archive ouverte pluridisciplinaire HAL, est destinée au dépôt et à la diffusion de documents scientifiques de niveau recherche, publiés ou non, émanant des établissements d'enseignement et de recherche français ou étrangers, des laboratoires publics ou privés. 


\title{
Flexible hydrofoil optimization for the $35^{\text {th }}$ America's Cup with constrained EGO method
}

\author{
Matthieu Sacher ${ }^{\mathrm{a}, *}$, Mathieu Durand ${ }^{\mathrm{b}, \mathrm{c}, \mathrm{d}}$, Élisa Berrini ${ }^{\mathrm{e}, \mathrm{f}}$, Frédéric Hauville ${ }^{\mathrm{a}}$, \\ Régis Duvigneau ${ }^{\mathrm{e}}$, Olivier Le Maître ${ }^{\mathrm{g}}$, Jacques-André Astolfi ${ }^{\mathrm{a}}$ \\ ${ }^{a}$ Naval Academy Research Institute - IRENAV CC600, 29240 BREST Cedex 9, France \\ ${ }^{b}$ K-Epsilon, 1300 Route des Crêtes, 06560 Valbonne, France \\ ${ }^{c}$ Groupama Team France, Lorient La Base - Batiment des défis, 56100 Lorient, France \\ ${ }^{d}$ Sirli Innovations, 600 Chemin des Aspres, 06410 Biot, France \\ e Université Côte d'Azur, Inria, CNRS, LJAD, 2004 Route des Lucioles, 06902 Valbonne, France \\ ${ }^{f}$ MyCFD, 29 Avenue des Frères Roustan, 06220 Golfe-Juan, France \\ ${ }^{g}$ LIMSI - CNRS, Rue John Von Neumann, 91400 Orsay, France
}

\begin{abstract}
This paper investigates the use of constrained surrogate models to solve the multi-design optimization problem of a flexible hydrofoil. The surrogate-based optimization (EGO) substitutes the complex objective function of the problem by an easily evaluable model, constructed from a limited number of computations at carefully selected design points. Associated with ad-hoc statistical strategies to propose optimum candidates within the estimated feasible domain, EGO enables the resolution of complex optimization problems. In this work, we rely on Gaussian processes (GP) to model the objective function and adopt a probabilistic classification method to treat non-explicit inequality constraints and non-explicit representation of the feasible domain. This procedure is applied to the design of the shape and the elastic characteristics of a hydrofoil equipped with deformable elements providing flexibility to the trailing edge. The optimization concerns the minimization of the hydrofoil drag while ensuring a non-cavitating flow, at selected sailing conditions (boat speed and lifting force). The drag value and cavitation criterion are determined by solving a two-dimensional nonlinear fluid-structure interaction problem, based on a static vortex lattice method with viscous boundary layer equations, for the flow, and a nonlinear elasticity solver for the deformations of the elastic components of the foil. We compare the optimized flexible hydrofoil with a rigid foil geometrically optimized for the same sailing conditions. This comparison highlights the hydrodynamical advantages brought by the flexibility: a reduction of the drag over a large range of boat speeds, less susceptibility to cavitation and a smaller angle of attack tuning range.
\end{abstract}

Keywords: Constrained optimization, Gaussian process model, hydrofoil, cavitation, fluid-structure interaction

\section{Introduction}

Created in 1851, the America's Cup trophy is one of the oldest international sports competition. The race opposes two sailing boats, representing a defender and a challenger yacht clubs, that race one against the other to be the winner of the America's Cup match races. In each edition, specific rules are provided by the defender in accordance with the Deed of Gift, which define the format of the regatta, the location of the races and restrictions on the type of boats allowed to compete. When a challenging yacht club wins the match races, it becomes the defender and gains the stewardship for the next America's Cup edition. The $35^{\text {th }}$ edition, which took place in June 2017, has been raced on so-called AC50s catamarans with size fixed to 50 feet, equipped with hydrofoils and wing-sails. The hydrofoils are expected to be a key component of the AC50s performance, and the competing teams are dedicating a growing fraction of their design effort to their optimization in comparison to older yacht design approach [Richards et al. 2001]. The

\footnotetext{
*Corresponding author

Email address: matthieu. sacher@ecole-navale.fr (Matthieu Sacher)
}

optimization aims at designing hydrofoil with a minimal drag force over a whole range of boat speeds (BS), while ensuring a sufficient lift force for the boat to fly, especially at the lowest $\mathrm{BS}$. The design of the hydrofoil is restricted by the "AC Class Rule" documents. In particular, the rule number 11.11 states: "Daggerboard components shall be rigidly fixed to each other and the daggerboard shape shall not be adjusted while racing." One possible way to comply with this rule, while allowing for some adaptivity of the shape with the BS, is to design a hydrofoil composed of rigidly mounted components, undergoing large elastic deformations under the hydrodynamical loads and without crew adjustments [Hueber et al. 2017]. For instance, a reduction of the hydrofoil camber, with increasing BS and hydrodynamic loads, can be obtained using a flexible trailing edge.

Drag reduction by shape optimization is an important research topic, with many applications in domains ranging from aeronautic to wind-energy recovery. Multiple studies have been proposed, based on physical models with different levels of complexity. For instance, the geometrical optimization of an airfoil with a two-dimensional steady flow model is considered in Ribeiro et al. [2012] when the unsteady flow situation 
is treated in Srinath and Mittal [2010]. Complex optimizations for a range of Mach numbers of a two-dimensional airfoil have been performed by $\mathrm{Li}$ et al. [2002], and the robust optimization for uncertain flows and geometries is considered in Papadimitriou and Papadimitriou [2016]. The numerical optimization of hydrofoils is to our knowledge much rarer, and the hydrofoil literature generally focuses on the stability [Inukai et al. 2001], wake energy reduction [Kandasamy et al. 2011], and free surface elevation [Duvigneau and Visonneau 2004] aspects.

This paper reports the development and application, in partnership with the Groupama Team France (GTF) design team, of an Efficient Global Optimization (EGO) procedure tailored to the flexible hydrofoil optimization problem. We restrict ourselves to the optimization of a two-dimensional hydrofoil consisting of rigid forward and trailing parts, connected by a deformable element. The objective of the optimization is the reduction of the hydrodynamic drag at several BS and lift conditions, with cavitation constraints. Cavitation is indeed an important concern for the hydrofoil performance [Sedlar et al. 2016], but its numerical prediction remains a difficult problem, as shown for instance in Leroux et al. [2005], Coutier-Delgosha et al. [2007], Ducoin et al. [2009], Akcabay et al. [2014]. These difficulties explain that cavitation aspects are usually not considered in hydrofoil shape optimization, unless when the objective is precisely to delay the cavitation, such as in WEI et al. [2015].

The optimization of flexible hydrofoils raises many challenges. First it requires multi-design variables to prescribe the foil geometry at rest (unloaded shape), on the one hand, and the elastic properties and the internal structure of the deformable parts, on the other hand. Second, the evaluation of the performance of a given design requires the resolution of a fully nonlinear fluid-structure interaction (FSI) problem, possibly combining different boat speeds, with a numerical cost that inherently limits the number of designs that can be evaluated. Finally, for a given design, the consideration of non-cavitating flow requires an appropriate treatment of constraints that are costly to evaluate. As a matter of fact, the numerical optimization requires dedicated techniques to circumvent the computational complexity of the problem. In the present work, the numerical modeling involves the coupling of the two-dimensional vortex lattice method solver XFOIL [Drela 1989], to compute the flow, with the structural ARA software developed by KEpsilon [Durand et al. 2014], to compute the hydrofoil deformations. Even for this two-dimensional modeling, the computational times prevent the direct optimization based on the FSI solution with standard methods.

We then rely on a surrogate model to reduce the computational burden of optimizing complex systems with costly objective function estimation [Simpson et al. 2001]. Specifically, we use Gaussian processes [Kleijnen 2009] surrogate models with Efficient Global Optimization (EGO) strategies [Jones et al. 1998], that have been previously applied to aerodynamic drag reduction [Jeong et al. 2005], vibration reduction for rotating aircrafts [Glaz et al. 2009], optimization of FSI problems [Aghajari and Schäfer 2015] and sail trimming optimization [Sacher et al. 2017]. The classification approach for discontinuous or binary constraints proposed in Basudhar et al. [2012] is extended to the treatment of the cavitation constraints in the optimization procedure. In our approach, The admissible domain is approximated using Least-Squares Support Vector Machine (LS-SVM) [Suykens and Vandewalle 1999] regression from the previous observations. An original contribution of the work is a new probabilistic treatment of the constraints which is designed to mitigate the risk of exploring unfeasible solutions (which yield a cavitating flow).

This optimization procedure used in the present work is briefly described in Section 2, while Section 3 discusses the formulation of the optimization problem for the flexible hydrofoil, including the definition of the optimization variables and a brief discussion of the numerical models. Section 4 presents the optimization results, emphasizing on impact of the flexibility of the performance of the optimal hydrofoil. In particular, these performances are contrasted with the case of a rigid hydrofoil optimized for the same conditions. We have tried to provide as much as possible quantitative results, but for confidentiality reasons related to the partnership with the GTF design team, some values can not be provided and only trends are reported. Finally, conclusions and recommendations for future development are given in Section 5.

\section{GP-based constrained optimization}

The objective is to estimate the solution $\mathbf{x}_{\mathrm{opt}}$ of the following abstract optimization problem

$$
\min _{\mathbf{x} \in \Omega} \mathcal{P}(\mathbf{x}), \quad \text { s.t. } \quad \mathbf{Q}(\mathbf{x}) \geq \mathbf{0},
$$

where $\mathbf{x} \in \mathbb{R}^{d}$ is the vector of control variables, $\Omega$ the optimization domain, $\mathcal{P}: \Omega \mapsto \mathbb{R}$ the objective function and $\mathbf{Q}: \mathbf{x} \mapsto \mathbb{R}^{m}$ is the vector of constraints. Even in the unconstrained case, $m=0$, finding the global optimum of $\mathcal{P}$ can be very costly, in particular when its evaluation is numerically expensive. The use of surrogate models for $\mathcal{P}$ is then a classical solution to reduce the computational burden of complex systems optimization [Simpson et al. 2001]. We use Gaussian processes (GP) [Kleijnen 2009] which, owing to their statistical nature, provide both a prediction of the objective function and a measure of the uncertainty (variance) in the prediction. These features are appealing in optimization, as they can be used to derive rigorous optimization strategies based on the maximization of the Expected Improvement (EI) criterion, leading to methods referred globally as the Efficient Global Optimization (EGO) [Jones et al. 1998] methods. In Section 2.1 we review the construction of the GP model for $\mathcal{P}$ and the resulting optimization strategy in the unconstrained case. The constrained case is considered in Section 2.2.

\subsection{Unconstrained EGO using GP}

Consider a set of $n$ training inputs points $\mathbf{X}_{n}=\left\{\mathbf{x}_{1}, \ldots, \mathbf{x}_{n}\right\}$, each associated to a noisy observation $y_{i}$ of the objective function. Specifically, it is assumed that $y_{i}=\mathcal{P}\left(\mathbf{x}_{i}\right)+\varepsilon_{i}$, where the $\varepsilon_{i}$ are Gaussian measurement noises, assumed for simplicity independent and identically distributed with variance $\sigma_{\epsilon}{ }^{2}$. The GP 
construction considers that $\mathcal{P}$ is a realization of a zero-mean multivariate Gaussian process with covariance function $C_{\mathcal{P}}$. In this work, we consider the multidimensional squared exponential covariance functions defined by

$$
C_{\mathcal{P}}\left(\mathbf{x}, \mathbf{x}^{\prime} ; \boldsymbol{\Theta}\right) \doteq \theta_{1} \prod_{i=1}^{d} \exp \left(\frac{-\left(x_{i}-x_{i}^{\prime}\right)^{2}}{2 l_{i}^{2}}\right)+\theta_{2},
$$

where $\boldsymbol{\Theta}=\left\{\theta_{1}, \theta_{2}, l_{1}, l_{2}, \ldots, l_{d}\right\}$ is a vector of hyper-parameters to be inferred from the observations. From the conditional rules of joint Gaussian distributions [Rasmussen and Williams 2006], the best prediction $\widehat{\mathcal{P}}_{n}(\mathbf{x})$ of $\mathcal{P}(\mathbf{x})$, i.e. the mean of $y$, and the prediction variance $\hat{\sigma}_{\mathcal{P}}^{2}(\mathbf{x})$ are given by

$$
\begin{aligned}
& \widehat{\mathcal{P}}_{n}(\mathbf{x})=\mathbf{k}^{\mathrm{T}}(\mathbf{x})\left(\mathbf{C}(\boldsymbol{\Theta})+\sigma_{\epsilon}{ }^{2} \mathbf{I}\right)^{-1} \mathbf{Y}_{n} \\
& \hat{\sigma}_{\mathcal{P}}^{2}(\mathbf{x})=\kappa(\mathbf{x})+\sigma_{\epsilon}{ }^{2}-\mathbf{k}^{\mathrm{T}}(\mathbf{x})\left(\mathbf{C}(\boldsymbol{\Theta})+\sigma_{\epsilon}{ }^{2} \mathbf{I}\right)^{-1} \mathbf{k}(\mathbf{x}) .
\end{aligned}
$$

where $\mathbf{C}(\boldsymbol{\Theta}) \in \mathbb{R}^{n \times n}$ is the covariance matrix with entries $C_{i, j}(\boldsymbol{\Theta})$ $\doteq C_{\mathcal{P}}\left(\mathbf{x}_{i}, \mathbf{x}_{j} ; \boldsymbol{\Theta}\right), 1 \leq i, j \leq n, \kappa(\mathbf{x}) \doteq C_{\mathcal{P}}(\mathbf{x}, \mathbf{x} ; \boldsymbol{\Theta}), \mathbf{k}(\mathbf{x})$ is the covariance vector between the observations in $\mathbf{X}_{n}$ and $\mathbf{x}$, and $\mathbf{I}$ is the identity matrix of $\mathbb{R}^{n}$. The hyper-parameters $\boldsymbol{\Theta}$ and noise variance $\sigma_{\epsilon}^{2}$ can be determined by maximizing the logmarginal likelihood [Rasmussen and Williams 2006] using an evolution strategy algorithm [Hansen 2006]. More details on GP meta-models can be found in [Rasmussen and Williams 2006].

Let $\hat{\mathbf{x}}_{n}$ be the optimum of $\widehat{\mathcal{P}}_{n}(\mathbf{x})$. It is expected that $\hat{\mathbf{x}}_{n} \approx \mathbf{x}_{\text {opt }}$ if the approximation error $\widehat{\mathcal{P}}_{n}-\mathcal{P}$ is small enough. The advantage of minimizing $\widehat{\mathcal{P}}_{n}$ instead of $\mathcal{P}$ is that GP models are usually inexpensive to evaluate compared to the original objective function. To control the error in the approximation, one proceeds sequentially by adding progressively new observation points in the area of interest. A deterministic optimization procedure would choose the next point $\mathbf{x}_{n+1}$ as the optimal point of $\widehat{\mathcal{P}}_{n}$. However, the GP model provides probabilistic information that can be exploited to propose more robust strategies based on merit functions, which combine the prediction and its variance. In this work, we use the Augmented Expected Improvement (AEI) merit function [Huang et al. 2006], which estimates the expected progress in the objective, taking into account the noise in the observed values and the prediction variance:

$$
A E I(\mathbf{x})=E I(\mathbf{x})\left(1-\frac{\sigma_{\epsilon}}{\sqrt{\hat{\sigma}_{\mathcal{P}}^{2}(\mathbf{x})+\sigma_{\epsilon}^{2}}}\right),
$$

where the Expected Improvement $E I(\mathbf{x})$ is defined by

$$
\begin{aligned}
E I(\mathbf{x}) & =\hat{\sigma}_{\mathcal{P}}(\mathbf{x})[u(\mathbf{x}) \Phi(u(\mathbf{x}))+\phi(u(\mathbf{x}))], \\
u(\mathbf{x}) & =\frac{\widehat{\mathcal{P}}_{n}\left(\mathbf{x}_{*, n}\right)-\widehat{\mathcal{P}}_{n}(\mathbf{x})}{\hat{\sigma}_{\mathcal{P}}(\mathbf{x})},
\end{aligned}
$$

with $\Phi$ and $\phi$ the cumulative and density functions of the standard Gaussian distribution, and $\mathbf{x}_{*, n} \in \mathbf{X}_{n}$ is the current effective best solution (see [Huang et al. 2006]). The optimum $\mathbf{x}_{n+1}$ of the AEI is added to $\mathbf{X}_{n}$, and $\mathcal{P}$ is evaluated at the new point providing $y_{n+1}$. Setting $n \leftarrow n+1$, a new iteration can start updating the GP model with the new observation. Overall, each iteration requires one computation of the objective and the resolution of two optimization problems: a first one for the hyper-parameters of the GP model, and a second one to find the AEI optimum. The iterations of the GP-based optimization problem are continued until a stopping criterion is satisfied or the resources allocated to the optimization have been exhausted.

\subsection{EGO under constraints, a classification based approach}

EGO methods with inequality constraints was considered in Schonlau [1997]. The key idea was to rely on $m$ additional surrogates to estimate the constraints $Q_{i}(\mathbf{x})$. For Gaussian Process models, one can easily determine the probability $P_{i}\left(Q_{i} \geq\right.$ $0 \mid \mathbf{x})$ that a constraint $Q_{i}$ is satisfied at $\mathbf{x}$ and, assuming the independence of the constraints probability, to come up with the consolidated probability $P(\mathbf{Q} \geq \mathbf{0} \mid \mathbf{x})=\prod_{i=1}^{m} P_{i}\left(Q_{i} \geq 0 \mid \mathbf{x}\right)$. This probability is used to modify the unconstrained AEI criterion and favor feasible regions:

$$
A E I_{\mathbf{Q}}(\mathbf{x})=A E I(\mathbf{x}) P(\mathbf{Q} \geq \mathbf{0} \mid \mathbf{x}) .
$$

Although effective in many problems, this GP modeling of the constraints faces several limitations. First, its computational cost increases with the number $m$ of constraints and can be an issue for problems with large $m$. Second, the approximation by GP models assumes a sufficient smoothness of the $Q_{i}$, which must be evaluable for almost every $\mathbf{x} \in \Omega$. This rules out the case of binary constraints (feasible / infeasible) and models having no solution for $\mathbf{Q}(\mathbf{x}) \ngtr \mathbf{0}$. Finally, the approach is also limited to situations where the feasible domain has an explicit representation in terms of the constraints. The optimization of hydrofoils reaches some of these limitations as it is difficult to express feasible geometries in terms of constraints on design parameters, while some values may lead to uncomputable solutions.

Classification methods recently proposed in Basudhar et al. [2012] are better suited to deal with discontinuous or binary constraints in a GP-based optimization procedure. The binary classifier considers two classes $C^{+}$and $C^{-}$over $\Omega$, corresponding to the feasible and unfeasible domains respectively. Each $\mathbf{x}_{i}$ of $\mathbf{X}_{n}$ is equipped with a value $z_{i}= \pm 1$ depending on its membership $C^{ \pm}$. To predict the class of a new point $\mathbf{x}$ we introduce a classification function $h: \mathbf{x} \in \Omega \rightarrow \mathbb{R}$, such that $z(\mathbf{x})=\operatorname{sign} h(\mathbf{x})$. A Least-Squares Support Vector Machine (LSSVM) [Suykens and Vandewalle 1999] is used to construct $h$. The LS-SVM method extends the original Support Vector Machine (SVM) classifier Vapnik [1995] to quadratic penalization, resulting in a linear (but non-sparse) system to be solved (see below). Further details and discussion on SVM and LS-SVM methods can be found in Cawley [2006].

The LS-SVM method [Suykens and Vandewalle 1999] is a linear classifier, for $C^{+}$and $C^{-}$, in a feature space induced by the transformation $\phi: \Omega \rightarrow \mathcal{F}$ :

$$
h(\mathbf{x})=\mathbf{w}^{\mathrm{T}} \boldsymbol{\phi}(\mathbf{x})+b .
$$

Here, $\mathbf{w}$ and $\boldsymbol{\phi}(\mathbf{x})$ are the weights and features vectors, while $b \in \mathbb{R}$ is a constant. The feature space $\mathcal{F}$ is generated by a reproducing kernel $r: \Omega \times \Omega \rightarrow \mathbb{R}$ representing the inner product 
between images in $\mathcal{F}$ of vectors: $r\left(\mathbf{x}, \mathbf{x}^{\prime}\right)=\boldsymbol{\phi}(\mathbf{x}) \cdot \boldsymbol{\phi}\left(\mathbf{x}^{\prime}\right)$. We use the classical Gaussian kernel,

$$
r\left(\mathbf{x}, \mathbf{x}^{\prime}\right)=\exp \left(-\frac{\left\|\mathbf{x}-\mathbf{x}^{\prime}\right\|^{2}}{2 \lambda^{2}}\right),
$$

with scale factor $\lambda \in \mathbb{R}$ to be adjusted. The LS-SVM parameters $(\mathbf{w}, b)$ satisfy the primal constrained optimization problem

$$
\begin{array}{ll}
\min _{\mathbf{w}, b, \mathbf{e}} & \frac{1}{2}\|\mathbf{w}\|^{2}+\gamma \frac{1}{2} \sum_{i=1}^{n} e_{i}^{2}, \\
\text { s.t. } & z_{i}=\mathbf{w}^{\mathrm{T}} \boldsymbol{\phi}\left(\mathbf{x}_{i}\right)+b+e_{i}, \quad i=1, \ldots, n,
\end{array}
$$

with trade-off parameter $\gamma \in \mathbb{R}^{+}$(to be fixed) and relaxation variables $e_{i}$ allowing for miss-classification. By writing the Lagrangian of the optimal problem, we introduce the $\alpha_{i} \in \mathbb{R}$, that are the Lagrange multipliers of the constraints. Denoting $\mathbf{z}=\left(z_{1} \cdots z_{n}\right)^{\mathrm{T}}$, the optimality conditions of the Lagrangian are used to derive a linear system for the dual model parameters $b$ and $\alpha=\left(\alpha_{1} \cdots \alpha_{n}\right)^{\mathrm{T}}$,

$$
\left[\begin{array}{cc}
\mathbf{R}+\gamma^{-1} \mathbf{I} & \mathbf{1} \\
\mathbf{1}^{\mathrm{T}} & 0
\end{array}\right]\left[\begin{array}{l}
\boldsymbol{\alpha} \\
b
\end{array}\right]=\left[\begin{array}{l}
\mathbf{z} \\
0
\end{array}\right],
$$

where $\mathbf{R} \in \mathbb{R}^{n \times n}$ is the kernel matrix with $R_{i, j}=r\left(\mathbf{x}_{i}, \mathbf{x}_{j}\right)$. This system is solved via a Cholesky factorization [Cawley 2006], and $h$ in (7) is expressed in terms of dual model parameters to obtain

$$
h(\mathbf{x})=\sum_{i=1}^{n} \alpha_{i} r\left(\mathbf{x}_{i}, \mathbf{x}\right)+b .
$$

The LS-SVM classifier depends on two parameters, $\gamma$ and $\lambda$, to be fixed when solving (10). We rely on a Leave-One-Out procedure to determine $\gamma$ and $\lambda$ that minimize the Predicted Residual Sum-of-Squares (PRESS) criterion [Allen 1974]. A simple expression for the predicted residuals is proposed in Cawley and Talbot [2003], which is presently minimized for $\lambda$ and $\gamma$ with the CMA-ES algorithm [Hansen 2006].

Finally, the LS-SVM binary classifier is extended to a probabilistic classification, relating $h$ in (11) to the probability of the class $C^{+}$, denoted $P\left(C^{+} \mid \mathbf{x}\right)$. A comparison of several probability models for the LS-SVM classification is provided in Van Calster et al. [2007]. We use the sigmoid function [Platt 1999] and expressing the probability of $C^{+}$as

$$
P\left(C^{+} \mid \mathbf{x}\right)=\frac{1}{1+\exp (A h(\mathbf{x})+B)} .
$$

The parameters $A$ and $B$ of the sigmoid are determined by minimizing the probability of misclassification, see [Platt 1999, Lin et al. 2007]. In practice, the probability $P$ goes to 1 (resp. 0) as the classifier is certain that $\mathbf{x}$ is feasible and belongs to $C^{+}$ (resp. unfeasible and belongs to $C^{-}$), while a value of $P=1 / 2$ denotes a complete uncertainty in the classification. This can occur because $\mathbf{x}$ is far from any observations in $\mathbf{X}_{n}$ or close to the interface between the two classes.

Following [Schonlau 1997] and [Basudhar et al. 2012], the probability of feasibility / evaluability is used to derive from the
AEI a merit function and select a new point $\mathbf{x}_{n+1}$ that yields the highest expected improvement while having a high probability of feasibility / evaluability. To this end, Basudhar et al. [2012] uses a modified version of the sigmoid function in (12) that accounts for the distances to the closest classified observations (in $\mathbf{X}_{n}$ ). While improving the misclassification rate, this modification results in a discontinuous merit function which prevents the use of gradient-based optimization tools to determine $\mathbf{x}_{n+1}$. Further, their strategy requires the addition of $d+1$ new observation points per iteration (to maintain isotropy in the observation points distribution) with an associated cost deemed too important in our application. Instead, we use the original definition (12) to extend the unconstrained AEI and we consider a sequential infilling strategy that follows the EGO iterations, by selecting alternatively one of the present definitions for $\mathbf{x}_{n+1}$ :

$$
\begin{aligned}
& \mathbf{x}_{n+1}=\underset{\mathbf{x} \in \Omega}{\arg \max } A E I(\mathbf{x}) P\left(C^{+} \mid \mathbf{x}\right), \\
& \mathbf{x}_{n+1}=\underset{\mathbf{x} \in \Omega}{\arg \max } A E I(\mathbf{x}) \quad \text { s.t. } \quad P\left(C^{+} \mid \mathbf{x}\right) \geq \rho, \\
& \mathbf{x}_{n+1}=\underset{\mathbf{x} \in \Omega}{\arg \min } \widehat{\mathcal{P}}_{n}(\mathbf{x}) \quad \text { s.t. } \quad P\left(C^{+} \mid \mathbf{x}\right) \geq \rho, \\
& \mathbf{x}_{n+1}=\underset{\mathbf{x} \in \Omega}{\arg \max }\left[A E I(\mathbf{x}) P\left(C^{+} \mid \mathbf{x}\right)\left(1-P\left(C^{+} \mid \mathbf{x}\right)\right)\right] .
\end{aligned}
$$

The first definition in (13) corresponds to the extension of the AEI favoring points with high chance of feasibility. The second expression (14) maximizes the original AEI definition by enforcing a minimal probability $\rho$ of feasibility; we use $\rho=0.5$. In (15), the predicted cost is directly minimized and enforced by $\rho$. Finally (16) combines the feasibility and infeasibility probabilities to favor areas where the classification is the most uncertain $(P \sim 0.5)$, with the objective to improve the exploration of the feasible domain boundaries. In practice, the new points $\mathbf{x}_{n+1}$ are determined using CMA-ES algorithms without Hansen [2006] or with constraints Arnold and Hansen [2012], depending on the considered definition.

As a final note, we remark that the optimization procedure can generate points that are found unfeasible in the sense that $\mathcal{P}\left(\mathbf{x}_{n+1}\right)$ cannot be computed. This is typically the case when $\mathbf{x}_{n+1}$ corresponds to an impossible geometrical configuration, or to an extreme situation for which the numerical code is not able to converge. It that case, the point is deemed infeasible, setting $z_{n+1}=-1$, but no value of the objective function is provided and the construction of the GP model for $\mathcal{P}$ simply disregard the missing data. As a result, the GP model of $\mathcal{P}$ and the LS-SVM classification can involve different numbers of observations.

\section{Hydrofoil optimization setup}

This Section introduces the formulation of the constrained optimization problem for the flexible hydrofoil, as defined in collaboration with GTF. Note that some details of the optimization problem are omitted for confidentiality reasons. We also briefly discuss the fluid and structural solvers used in this work.

\subsection{Problem formulation}

The optimization of the hydrofoil uses control variables $\mathbf{x}$ which parameterize the geometry and of the hydrofoil at rest, 
i.e. in the absence of fluid loads, and the elastic properties of the element that gives flexibility to the trailing edge (see below). The optimization concerns the minimization of the hydrofoil drag force over a set of 4 sailing conditions, with an additional set of 4 constraints designed to prevent the cavitation of the flow. Each condition corresponds to a prescribed boat speed and an hydrodynamic lift force developed by the hydrofoil. Note that the 4 drag points and the 4 constraints correspond to different conditions so the evaluation of the objective function $\mathcal{P}$ and the vector of constraints $\mathbf{Q}$ at given $\mathbf{x}$ requires solving 8 fluid-structure interaction problems. The optimization problem is written as

$$
\begin{array}{ll}
\min _{\mathbf{x} \in \Omega} & \mathcal{P}(\mathbf{x})=\sum_{i=1}^{4} w_{i} \mathrm{C}_{\mathrm{d}_{\mathrm{i}}}(\mathbf{x}), \\
\text { s.t. } \quad & \left\{\begin{array}{l}
Q_{1}(\mathbf{x})=-\mathrm{Cp}_{\min _{1}}(\mathbf{x}) \leq \lambda_{1} \\
Q_{2}(\mathbf{x})=-\mathrm{C} \mathrm{p}_{\min _{2}}(\mathbf{x}) \leq \lambda_{2} \\
Q_{3}(\mathbf{x})=-\mathrm{Cp}_{\min _{3}}(\mathbf{x}) \leq \lambda_{3} \\
Q_{4}(\mathbf{x})=-\mathrm{Cp}_{\min _{4}}(\mathbf{x}) \leq \lambda_{4}
\end{array} .\right.
\end{array}
$$

Here, the $\mathrm{C}_{\mathrm{d}_{\mathrm{i}}}$ are the drag force coefficients, the $w_{i}>0$ are prescribed weights, the $C \mathrm{p}_{\min _{i}}$ are the minimum pressure coefficients, and the $\lambda_{i}$ are the cavitation numbers defined by

$$
\lambda=\frac{p_{0}-p_{v}}{\frac{1}{2} \rho U_{0}^{2}} .
$$

We have denoted $U_{0}$ and $p_{0}$ the reference velocity (that is, the boat-speed) and pressure, $\rho$ the fluid density, and $p_{v}$ the saturated vapor pressure. The reference pressure is taken as $p_{0}=$ $p_{\text {atm }}+\rho g h$, where $p_{\text {atm }}$ is the atmospheric pressure, $g$ is the gravity acceleration, and $h$ is the immersion depth of the hydrofoil. The minimum of the pressure coefficient is defined by $\mathrm{Cp}_{\min }=\frac{p_{\min }-p_{0}}{0.5 \rho U_{0}^{2}}$, where $p_{\min }$ is the minimal pressure over the hydrofoil surface. The simplest criterion to prevent cavitation is to ensure that the minimal pressure remains higher than the vapor pressure: $p_{\min } \geq p_{v}$. This condition can be expressed in terms of cavitation number $\lambda$ to obtain the conditions on the minimum pressure coefficient $\mathrm{Cp}_{\min }$ to formulate the constraints in (17).

\subsection{Hydrofoil parametrization}

The geometric and elastic characteristics of the flexible hydrofoil are defined starting from a baseline geometry provided by GTF. The baseline geometry was produced by an optimization procedure using an evolutionary method [Bäck and Schwefel 1993] and assuming a complete rigidity of the hydrofoil (no elastic effects). The geometry at rest of the flexible hydrofoil is defined by rotating the sections of the baseline geometry (rigid hydrofoil), without changing the sections thickness. We denote $\mathrm{p}(s)$ the law of the sections rotation angle, where $0 \leq s \leq 1$ is the reduced coordinate along the chord of the baseline geometry. In practice we restrict the rotation to the last $30 \%$ of the hydrofoil chord $(\mathrm{p}(s)=0, s \leq 0.7)$ and use a smooth B-Spline interpolation [Piegl and Tiller 1997, Wang et al. 2006] of $\mathrm{p}(s)$ between 4 control points $0.7<s_{1}<\cdots<s_{4}=1$, as illustrated in Figure 1. We shall refer to the rotation angles $p_{1, \ldots, 4}$ at the control points as the geometrical parameters.

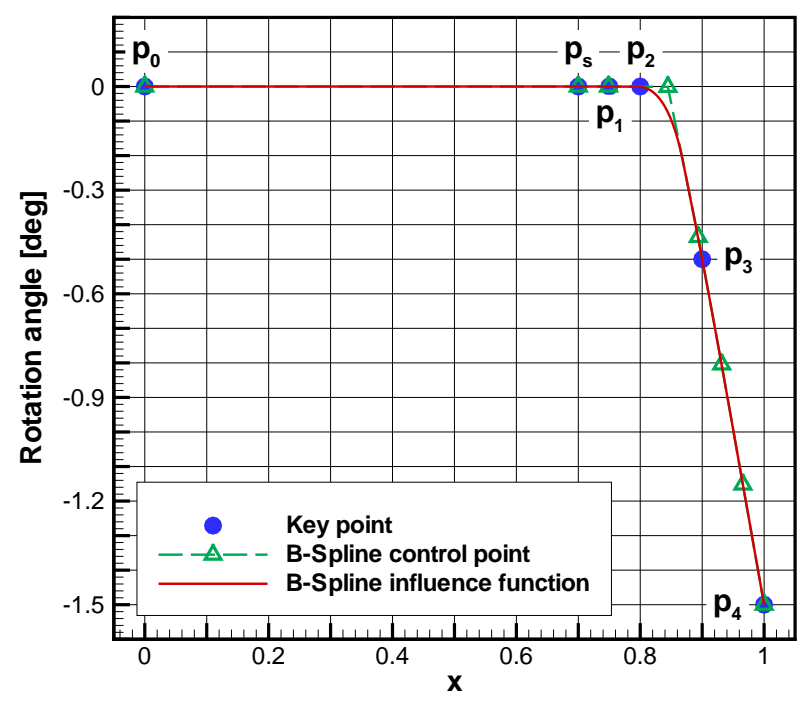

(a) B-Spline approximation of the rotation angle.

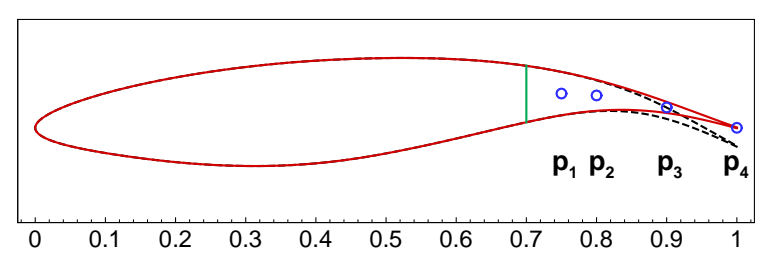

(b) Initial and deformed hydrofoils.

Figure 1: Illustration of the unloaded geometry definition using a rotation of the baseline geometry. The top plot shows the B-Spline approximation of the rotation rule $\mathrm{p}(s)$ based on the rotation angles $\mathrm{p}_{1}$ to $\mathrm{p}_{4}$ at the control points. The bottom plot compares the baseline (red) and unloaded flexible geometries, the green vertical line indicating the chord position $s=0.7$.

Regarding the flexibility of the hydrofoil, the results presented in the following consider a perfectly rigid hydrofoil except for an elastic element located in the backward part of the hydrofoil. As illustrated in Figure 2, the location of the elastic element is prescribed by the reduced coordinates of its starting and ending points $0<\mathrm{s}_{1}<\mathrm{s}_{2}<1$ (resp. $0<\mathrm{ds}_{1}<\mathrm{ds}_{2}<1$ ) on the lower (resp. upper) surface of the baseline hydrofoil. The deformable element is made of a homogeneous material having a Young modulus $\mathrm{E}_{\mathrm{LST}}$; it is further reinforced by a beam (shown in black in Figure 2) on the pressure surface (lower side). The elastic beam has a progressive Young modulus varying linearly between $s_{1}$ and $s_{2}$, with mean value $E_{B e a m}$ and slope $\mathrm{C}_{\text {Beam. }}$. The use of a single, homogeneous, flexible element is supported by preliminary optimization studies briefly presented in the Appendix at the end of the paper. Overall, the parametrization of the deformable part involves 7 parameters (4 geometric coordinates and 3 elastic properties) globally referred to as the structural parameters.

\subsection{Fluid-structure interaction solver}

Given a value of the geometric and structural parameters, the evaluation of the objective function and the constraints of the optimization problem calls for the resolution of 4 FSI problems (one for each condition of the objective function). We 


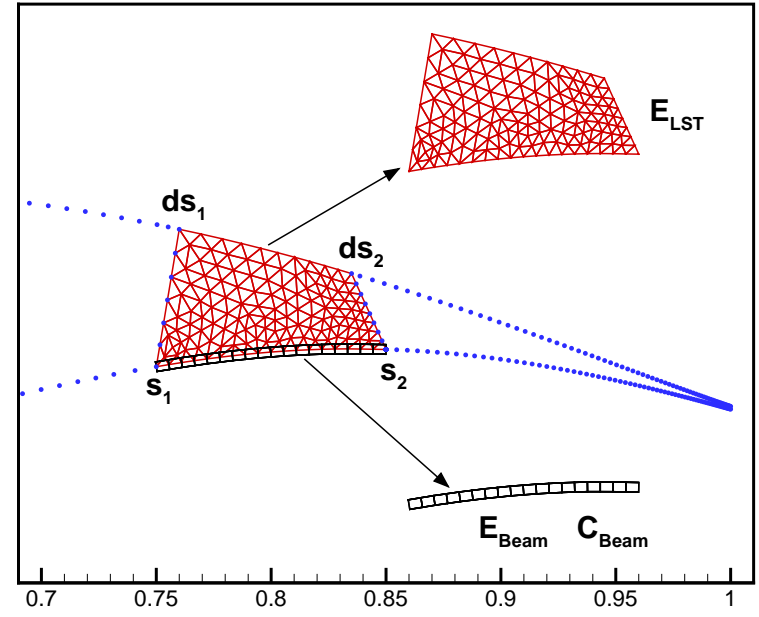

Figure 2: Illustration of the structural parameters.

assume that these problems have steady solutions for all values of the parameters and we rely on a quasi-steady approach to treat the coupling between the structural and fluid solvers.

For the flow solver, we rely on XFOIL [Drela 1989] which has been developed for many years and is widely used for many aerodynamic applications. While based on a two-dimensional airfoil panel code method, XFOIL can provide viscous and inviscid analyses. It incorporates a two-equation integral formulation of the viscous boundary layer and the approximate $e^{N}$ envelope method [Van Ingen 2008]; it allows the prediction of transition points and separation bubble, through the resolution of the boundary layer and the transition equations which is based on a Newton method. Further details on XFOIL are given in Drela [1989] and it is compared with other CFD methods and solvers in Morgado et al. [2016]. We only mention that the setting of our optimization problem imposes to enforce a prescribed lift force in the flow computation. This is achieved in XFOIL by determining the Angle of Attack (AoA) that yields the requested lift force. Overall, XFOIL is simple to use and has a reasonable computation cost on a classical workstation, in most of the cases, making it a natural candidate to be coupled with a structural solver (see for instance [MacPhee and Beyene 2013] for a recent FSI study of wind turbine blades using XFOIL).

The ARA software developed by K-Epsilon was used to solve the structural model of the flexible hydrofoil. The solver was originally developed for FSI computations of sails [Durand et al. 2014] and proposes various structural elements [Durand 2012]. In this work, the elastic part of the hydrofoil is modeled by 2D Linear Strain Triangles (LST) [Pedersen 1973] and Timoshenko beam elements (see Figure 2). The nonlinear equilibrium solution is computed by a Newton method with Aitken relaxation.

A quasi-monolithic algorithm [Durand 2012] is finally applied to couple the structural and flow solvers. Briefly, in this algorithm, the resolution of the structural problem is nested inside the iterations of the nonlinear steady flow solver. This ap- proach preserves the convergence rate and stability properties of the monolithic approach. The resulting coupled solver is finally driven by a utility that computes the set of FSI solutions required to estimate the objective function value and its constraints (17). This utility is itself nested into the optimization driver that decides of the sequence of new optimal parameters $\mathbf{x}_{n+1}$ to be evaluated.

\section{Results and discussions}

We now present the results of the optimization problem. In particular, we contrast the optimal flexible hydrofoil with its rigid counterpart optimized for the same set of conditions and constraints. This comparison concerns the characteristics and performances of the two optimal hydrofoils. We do not provide a comparison in terms of computational times because the two optimizations use different strategies and have significantly different complexities. Indeed, the optimization of the rigid hydrofoil although involving a larger number of optimization variables has an objective function and constraints much faster to compute than in the flexible case (which requires the resolution of FSI problems), enabling the application of evolutionarybased methods [Bäck and Schwefel 1993].

In the following, the rigid hydrofoil corresponds to the optimized baseline geometry provided by GTF, assuming a perfectly rigid hydrofoil, while the unloaded geometry refers to the rest geometry resulting from our optimization of the flexible hydrofoil, that is, the rigid geometry with the rotations of the trailing edge sections (but without elastic deformations). We stress that the structural and geometrical parameters characterizing the flexible hydrofoil and are not changing with the boat speed. Thus, the rigid, unloaded, and in fact, all flexible geometries are coinciding if the elastic element is infinitely stiff, because the rigid geometry is optimal in the absence of elastic deformations. For a finite elastic stiffness, one needs, in general, to jointly optimize the geometrical and structural parameters of the flexible hydrofoil to improve the performances compared to the rigid case. Optimizing only for the structural (resp. geometrical) parameters usually leads to a degradation of the performance, compared to the rigid hydrofoil case, unless the geometrical (resp. structural) parameters are fixed close to their joint optimization solution values.

\subsection{Selection of the design variables}

The computational time for the optimization of the flexible hydrofoil is an important aspect, and several tests were made to determine an appropriate trade-off between the computational and parametrization complexities, before arriving at the set-up described in Section 3.2 with the up to 11 parameters to be optimized ( 4 geometrical and 7 structural). The number of optimization variables can be further reduced, fixing, for instance, the material properties, the geometry of the deformable element, or changing the number of control points in the parametrization of the rotation rule. These reductions yield different complexities of the optimization problem with different computational times as a result. However, reducing 
the number of optimization variables also impacts the performance of the optimal design if the fixed parameters are not set close to their optimal values. Figure 3 shows the evolutions with the computational time of the estimated minimum of the objective function for different numbers of optimization variables considered in the optimization problem. The plot shows that when nine variables are considered the optimization needs $\approx 16$ times more resources to converge than for only five optimization variables, with close optimal objective values in this case. The case of two optimization variables is even less computationally demanding, but leads to a noticeable drop in the optimal performance because of inappropriately set values for the parameters disregarded in the optimization problem. These

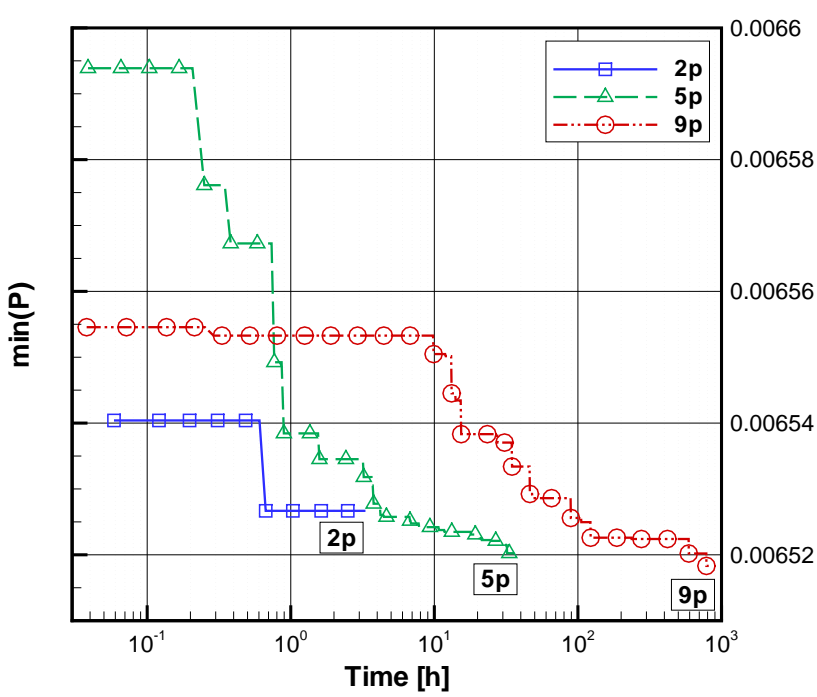

Figure 3: Convergence of the objective function minimum with the computational time, for different numbers of optimization variables considered.

effects can be better appreciated from Table 1 which reports the objective function values and associated computational times for the different numbers of design variables to optimize. This underlines the importance of identifying the most influent variables of the optimization problem. In the following, we focus

\begin{tabular}{c|cc} 
Dimension & $\min \mathcal{P}$ & Time $[\mathrm{h}]$ \\
\hline 2 & 0.006527 & 3 \\
5 & 0.006520 & 36 \\
9 & 0.006518 & 844
\end{tabular}

Table 1: Best objective function values, at convergence, and corresponding computational times for different numbers of optimization variables.

on the case of 9 optimization variables, corresponding to the 4 geometrical and 5 of the 7 structural parameters. $E_{B e a m}$ and $\mathrm{C}_{\mathrm{Beam}}$ have been fixed close to their optimization solution values.

\subsection{Optimal shapes and pressure distributions}

Figures 4 and 5 show for 20 and 40 kts of boat-speed respectively, the shapes of the rigid and flexible hydrofoils. These shapes are depicted at their effective angle of attack giving the prescribed lift force. The pressure coefficient distributions are also reported in the top part of the Figures. At low boat-speed (20 kts), in Figure 4, the rigid and deformed hydrofoils have noticeable differences in term of angle of attack: the rigid one is higher. Regarding the pressure coefficient distributions, the rigid case presents a minimum at the leading edge of $\approx-2.25$, whereas in the flexible case the minimum peaks to $\mathrm{Cp} \approx-1.1$ only, at the same location. This can be explained as a less cambered geometry generally requires a higher angle of attack to achieve the same lift force, while the minimum pressure coefficient usually increases with the angle of attack. Anyway, the results indicate a poorer tolerance to cavitation for the rigid hydrofoil in these conditions.

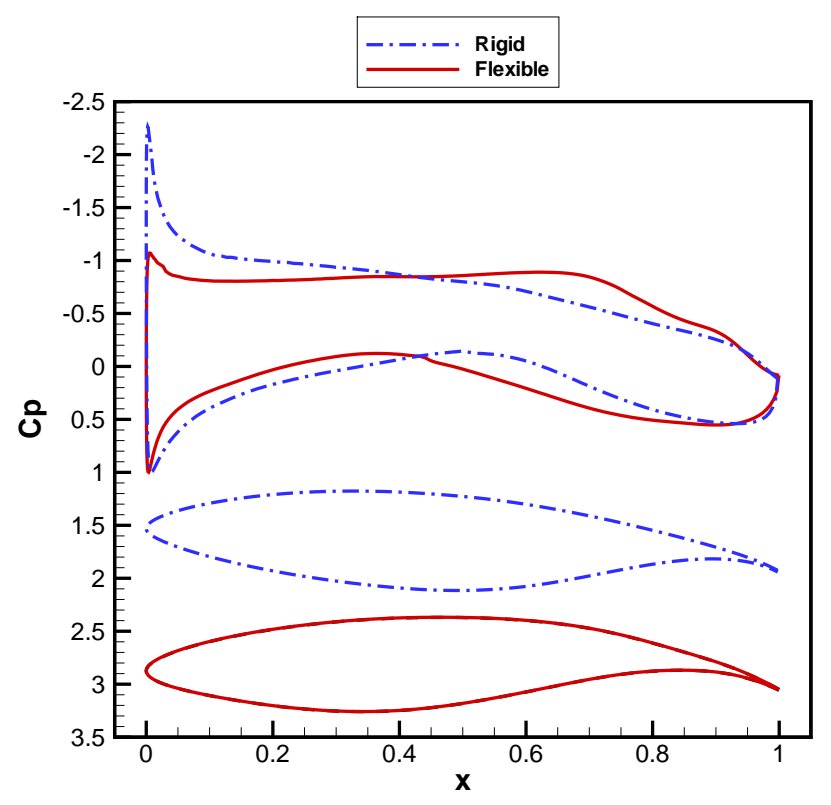

Figure 4: Shapes and distributions of $\mathrm{Cp}$ at $20 \mathrm{kts}$ of boat-speed for the rigid and flexible hydrofoils.

For 40 kts of boat-speed, shown in Figure 5, the two angle of attack are negative and the rigid one presents again the largest deviation from zero angle of attack. The deformation of the trailing edge is now pronounced, in the flexible case, as it can be appreciated using dashed black contour which corresponds to the unloaded flexible geometry (which comes from the GTF optimizations with flap laws). The trailing edge deflection is $\approx 9.5 \mathrm{deg}$. The minimum pressure coefficients are now located approximately at mid-chord with $C \mathrm{p}_{\min } \approx-0.5$ in the two cases. The distortions in the pressure coefficient distribution observed at $\approx 0.8$ percent of the chord in the case of the flexible hydrofoil are due to the transitions between the rigid parts and the deformable element constituting the flexible hydrofoil.

The flow around the flexible hydrofoil at $40 \mathrm{kts}$ of boatspeed has also been computed with a URANS transition model in order to validate the pressure coefficient distribution predicted by XFOIL. For that purpose, a structured mesh of the fluid domain around the hydrofoil has been generated using $\approx 110000$ elements; the flow was subsequently solved using 


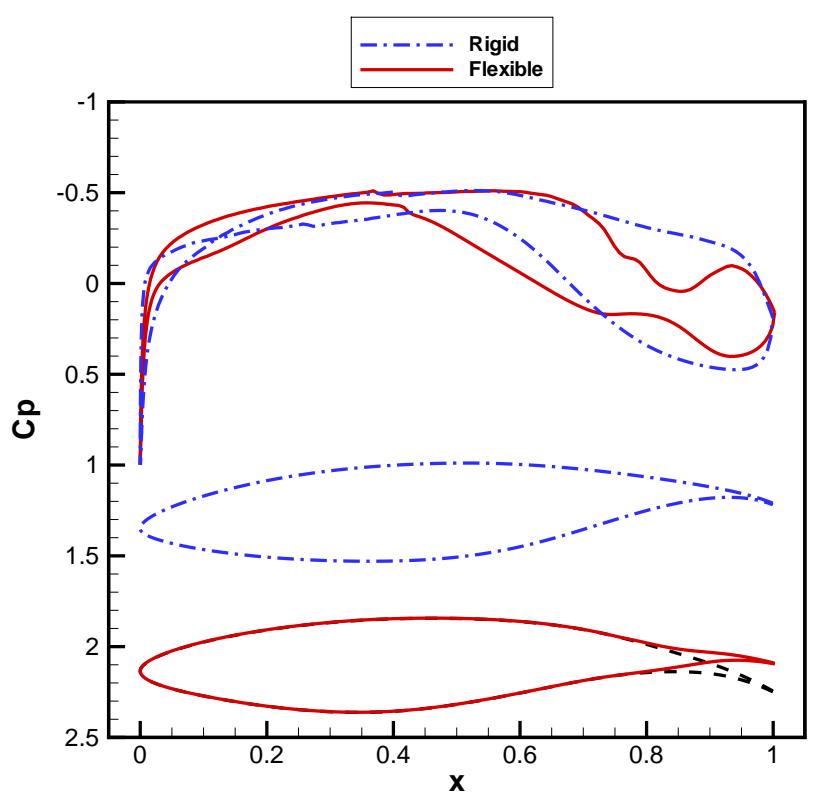

Figure 5: Shapes and distributions of the pressure coefficient $\mathrm{Cp}$ at $40 \mathrm{kts}$ of boat-speed for the rigid and flexible hydrofoils.

FLUENT with the Transition SST turbulence model [Menter et al. 2006]. The computed pressure coefficient distribution is reported in Figure 6, together with the XFOIL prediction, highlighting the excellent agreement between XFOIL and the Transition SST flow model. Small differences in the pressure coefficients are visible only on the pressure side at $\approx 90 \%$ of the chord length, and the magnitude of the differences in the global hydrodynamic loads are $2.6 \%$ and $4.2 \%$ for the lift and drag coefficients respectively. Thus, the pressure coefficients computed by XFOIL can be considered reliable, even in the area of the deformable element.

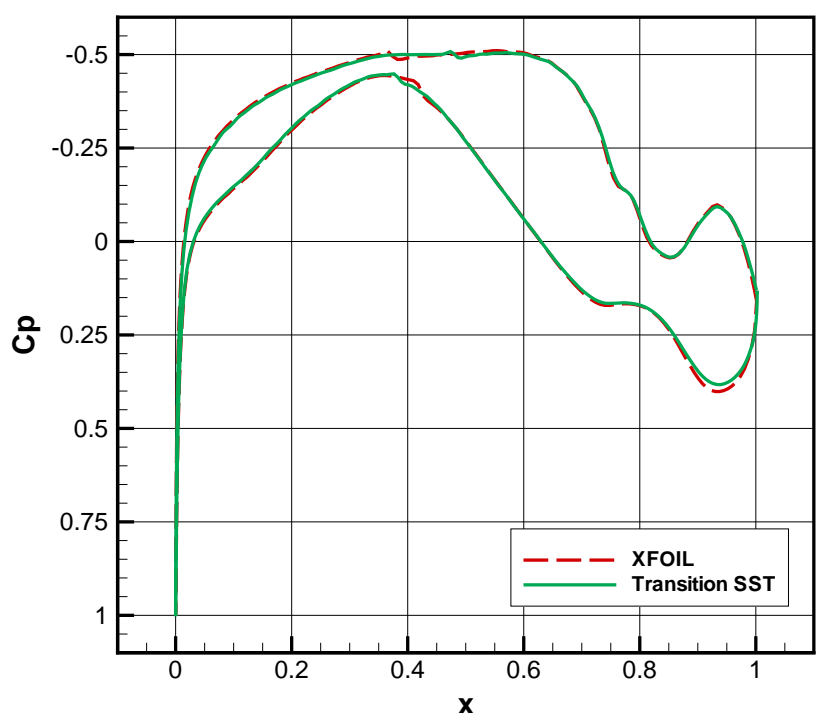

Figure 6: Distributions of the pressure coefficients Cp predicted by XFOIL and with the URANS transition models.
Figure 7 shows the evolutions of the camber along the chord for the rigid, unloaded and the loaded flexible hydrofoils at several boat-speeds. These geometrical quantities are computed based on the distances in the direction normal to the deformed chord, following the classical conventions. Note that because of the changes of the displacement of the trailing edge and the change in chord length with the boat-speed, for the flexible hydrofoil, the camber law in the forward part before the flexible element is also affected. The flexibility is seen to have an important effect for a boat-speed larger than $20 \mathrm{kts}$. In addition, the shapes of the camber distribution of the flexible hydrofoil differ significantly from the rigid one for all the boat-speeds shown. Specifically, the maximum camber of the flexible foil at $20 \mathrm{kts}$ of boat-speed is more than twice that of the rigid one, while at $40 \mathrm{kts}$ of boat-speed it is less by roughly $20 \%$. These evolutions of the apparent camber distribution with the boatspeed (and load in general) is one of the hydrodynamical advantages brought by the flexibility.

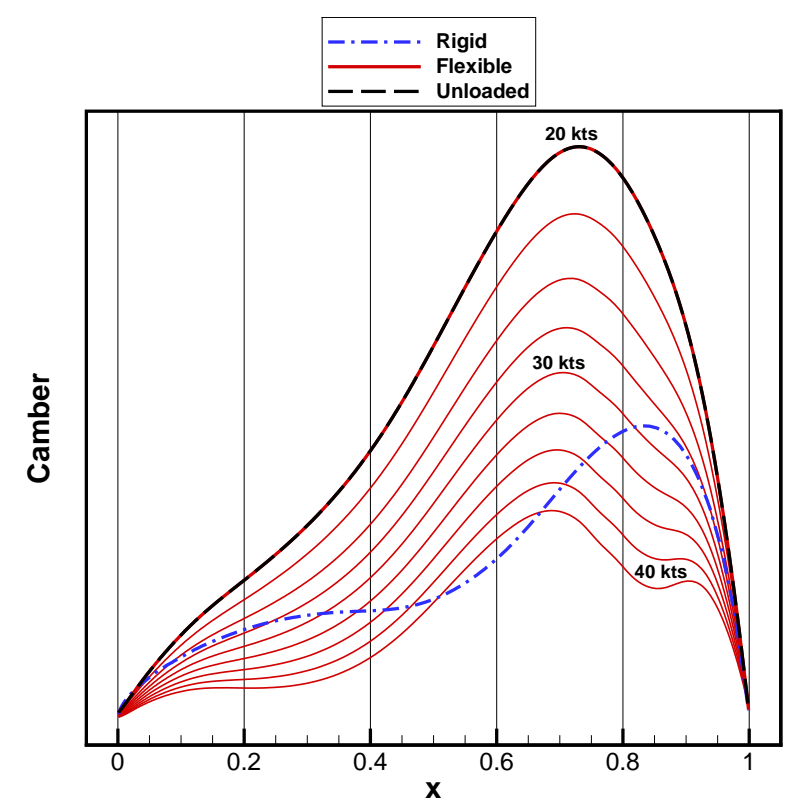

Figure 7: Flexible hydrofoil camber distribution at several boat-speeds. The rigid hydrofoil case is also shown for comparison.

\subsection{Angles of attack and cavitation criterion}

The effective angle of attack of the optimal hydrofoils is reported in Figure 8 as a function of the boat-speed. The previous observations regarding the angle of attack are confirmed. The angle of attack of the rigid hydrofoil almost linearly decreases from $\approx 3.9 \mathrm{deg}$ at $20 \mathrm{kts}$ to $\approx-0.9 \mathrm{deg}$ at $30 \mathrm{kts}$ of boat-speed, and subsequently continues to decrease, but at a lower rate, when the boat-speed increases further. For the flexible case, the maximum of angle of attack is only $\approx 1.8 \mathrm{deg}$ at $20 \mathrm{kts}$ and decreases to $\approx-0.1 \mathrm{deg}$ at $25 \mathrm{kts}$ of boat-speed. Then, unlike in the rigid case, the flexible hydrofoil keeps an almost constant effective angle of attack between 0 and $-1 \mathrm{deg}$ for the rest of boat-speed range, instead of decreasing continuously in the rigid case. Again, the flexibility can explain this 
behavior, as the deformation of the trailing edge (and reduction of the camber) discharges the foil when the boat-speed increases. This discharge calls for fewer changes in the angle of attack compared to the rigid case. Note that this sort of automatic trimming, through flexibility, may also be beneficial by saving some trimming efforts to the crew members.

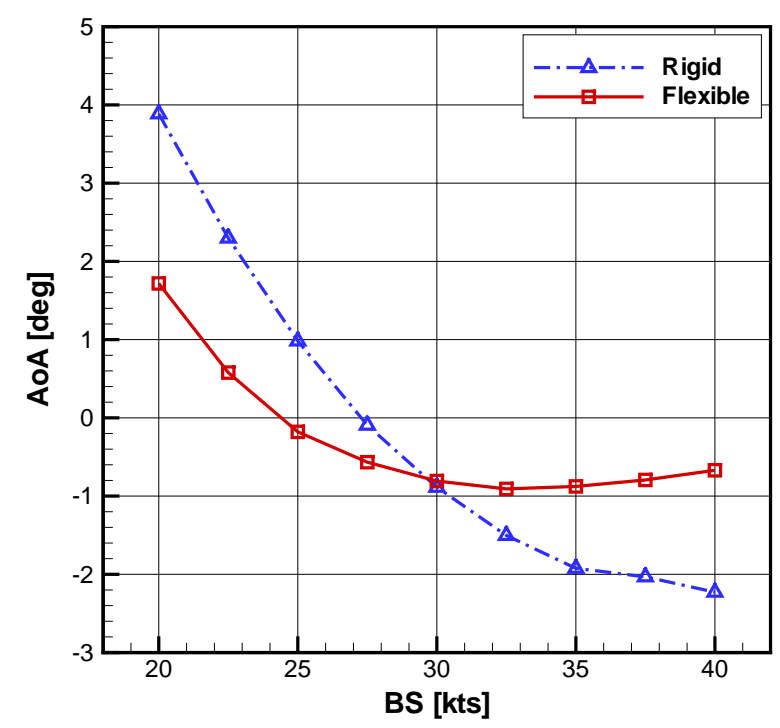

Figure 8: Effective angle of attack as a function of the boat-speed (BS).

Another positive impact of the flexible hydrofoil can be appreciated in Figure 9 which reports the minimum pressure coefficients for the two optimal hydrofoils, as a function of the boat-speed. The dashed black curve corresponds to the critical value based on the cavitation number (18). We thus expect the optimal hydrofoils to have a minimum pressure coefficient greater than this critical value over the whole range of boatspeeds considered. However, we recall that the cavitation criterion is enforced for a finite set of only 4 distinct values of the boat-speed. For the maximal boat-speed shown, $40 \mathrm{kts}$, the two optimal foils, in fact, violate by a small margin the criteria based on the cavitation number, which indeed is not explicitly enforced for this boat-speed. As lower boat-speeds are considered, till $25 \mathrm{kts}$, the two foils satisfy the non-cavitating criterion by a large margin, suggesting that any constraints in this range of boat-speeds would not be active. Finally, when the boatspeed approaches $20 \mathrm{kts}$ the behavior of the minimum pressure coefficient differs between the two hydrofoils: while for the flexible hydrofoil $\mathrm{Cp}_{\text {min }}$ remains away from its critical value, it decreases sharply and even becomes significantly lower than its critical value in the rigid case. Comparing the trends of the two hydrofoils, it can be concluded that the flexibility yields a design with minimum pressure coefficient much less dependent on the boat-speed, and therefore having lower chances of violating the non-cavitating conditions for a boat-speed other than in the constraints.

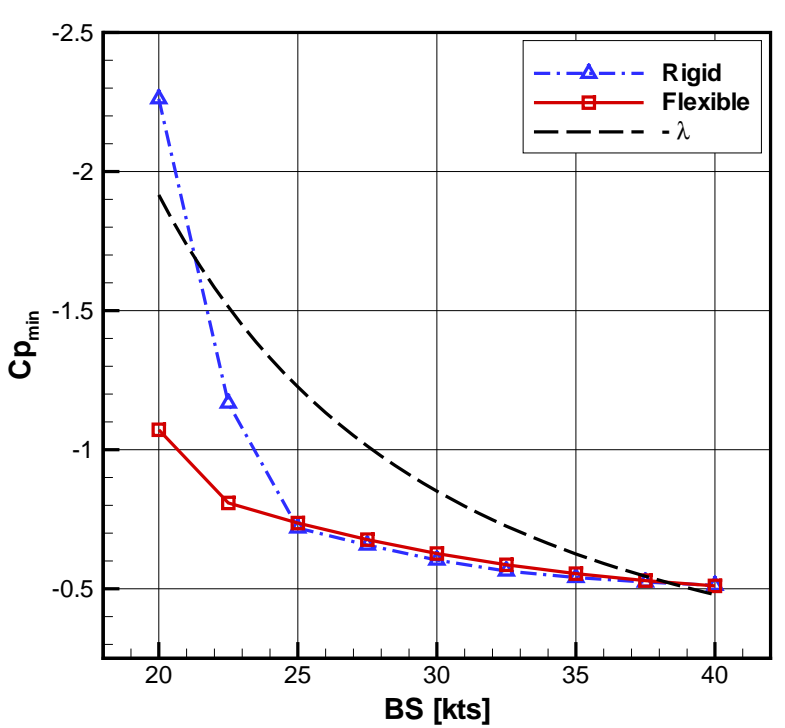

Figure 9: Minimum pressure coefficients $\mathrm{Cp}_{\min }$ as a function of the boat-speed (BS). Also shown is the cavitation number $\lambda$ given by (18).

\subsection{Drag performances}

To complete the comparison between the flexible and rigid hydrofoil, we report in Figure 10 the drag coefficients as a function of the boat-speed. The actual values are not shown, for confidentiality reasons, but the plot allows to appreciate the improvement brought by the flexible hydrofoil. Except for the lowest boat-speed (20 kts), the flexible hydrofoil has a drag coefficient $\mathrm{C}_{\mathrm{d}}$ consistently lower than for the rigid one. The improvement is particularly significant at 25 and 40 kts of boatspeed, with roughly $20 \%$ drag reduction at the highest boatspeed. Again, these improvements come from the flexibility which allows the hydrofoil to naturally adapt its camber with the boat-speed, with a thinner geometry at rest and lower variations of the angle of attack in the boat-speed range.

For a boat-speed between 20 and $25 \mathrm{kts}$, the hydrofoil drag is mainly depending on the location of the transition point on the suction side, denoted $\mathrm{x}_{\mathrm{tr}}$. As the boat-speed is lowered, the angle of attack increases to satisfy the required lift force and the transition point $\mathrm{x}_{\mathrm{tr}}$ moves toward the leading edge with a sharp increase in the friction drag (see Figure 10). Note that in XFOIL the location of the transition point is computed whenever it appears to be in the first $40 \%$ of the chord. Otherwise, it is set at this maximal location. To highlight the importance of the transition point on the drag of the hydrofoil, Figure 11 presents the evolutions of the flexible hydrofoil drag and the location of the transition point as functions of the boat-speed. The sharp drop in the hydrofoil drag, around $22.5 \mathrm{kts}$ of boat-speed, is clearly related to the displacement of the transition point away from the leading edge. Note that by using different weights and adding more conditions in (17), one could further improve the hydrofoil drag at a low boat-speed, by delaying the displacement of the transition point, but to the detriment of the drag at higher boat-speeds. 


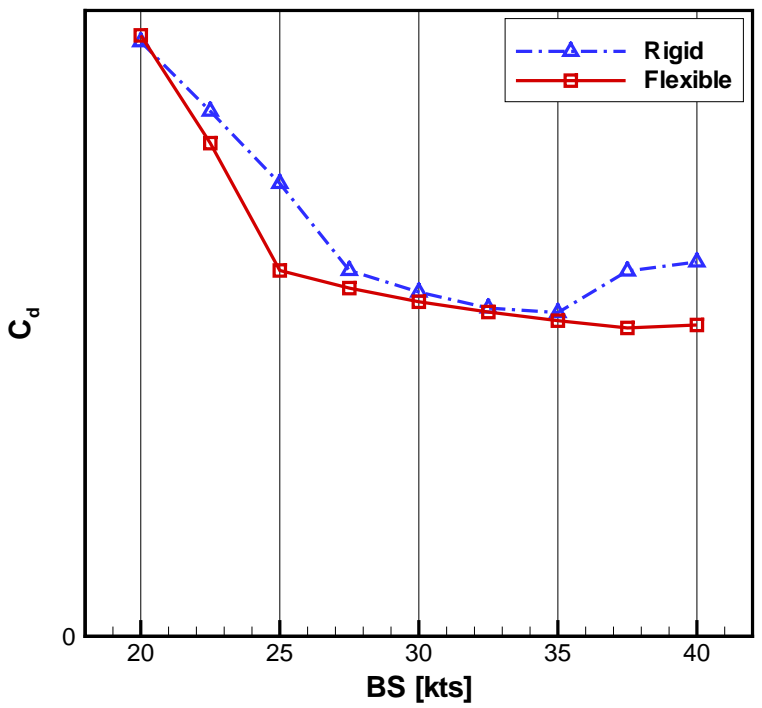

Figure 10: Drag $\mathrm{C}_{\mathrm{d}}$ of the optimal hydrofoils as a function of the boat-speed (BS).

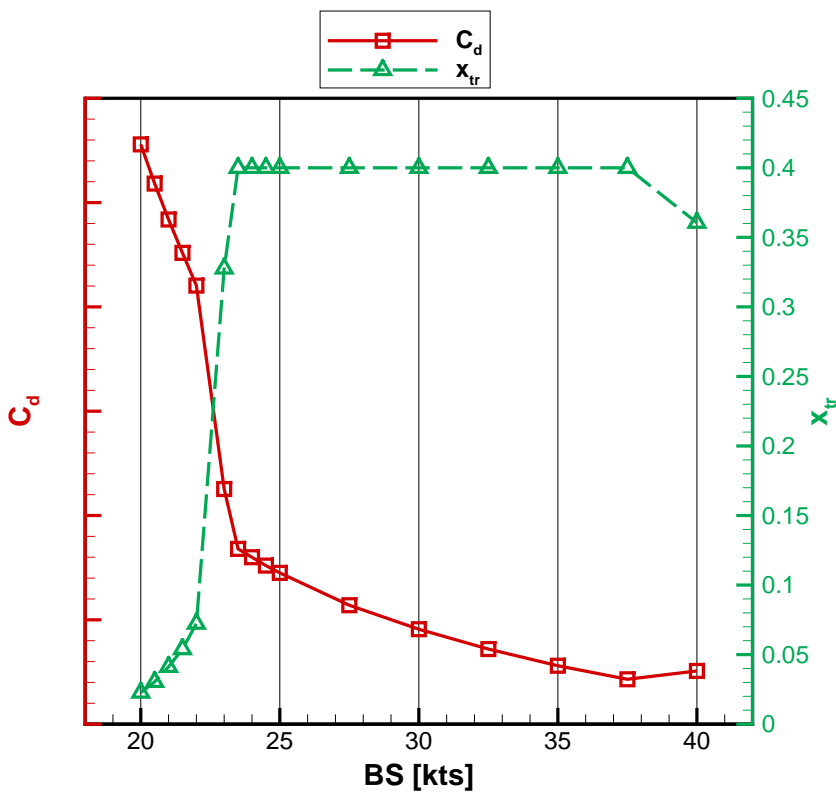

Figure 11: Drag $C_{d}$ and transition point location $x_{t r}$ for the flexible hydrofoil as functions of the boat-speed (BS).

\subsection{Optimal hydrofoil at $30 \mathrm{kts}$}

To illustrate the importance of the selected conditions used in the definition of the objective function, we optimize another rigid hydrofoil based on the minimization of the drag and noncavitating constraint in (17) based on the unique condition corresponding to $30 \mathrm{kts}$ of boat-speed. The design variables are reduced to the 4 geometrical angles $\mathrm{p}_{1, \ldots, 4}$ and we refer to this optimized hydrofoil as the Rigid 30 kts one.

Table 2 compares the angle of attack (AoA), minimum pressure coefficient $\mathrm{Cp}_{\min }$ and $\operatorname{drag} \mathrm{C}_{\mathrm{d}}$ of the flexible and Rigid 30 kts hydrofoils at $30 \mathrm{kts}$ of boat-speed. The Rigid $30 \mathrm{kts}$ case has a lower incidence, with a drag reduction of roughly $1.2 \%$ compared to the flexible case. The two $\mathrm{Cp}_{\text {min }}$ are relatively close and far from the criterion based on the cavitation number $\lambda$. The closeness of the two hydrofoils at this boat-speed of

\begin{tabular}{l|ccr} 
& Rigid 30 kts & Flexible & Difference \\
\hline AoA & $-0.36^{\circ}$ & $-0.81^{\circ}$ & 0.55 \\
$\mathrm{Cp}_{\min }$ & -0.605 & -0.627 & 0.035 \\
$\mathrm{C}_{\mathrm{d}}$ & - & - & -0.012
\end{tabular}

Table 2: Comparison of the hydrodynamical characteristics of the Rigid 30 kts and flexible solutions at $30 \mathrm{kts}$ of boat-speed.

$30 \mathrm{kts}$ can be further appreciated from Figure 12, where the two distributions of pressure coefficients $\mathrm{Cp}$ and the shapes plotted at the same angle of attack for comparison purposes are shown. The main difference in the pressure coefficient is the smoother character of the distribution in the Rigid $30 \mathrm{kts}$ case, which can be explained by the absence of the localized elastic deformations present in the flexible case. However, the differences between the two distributions are small as one could have expected from the similarity of the shapes and effective angle of attack.

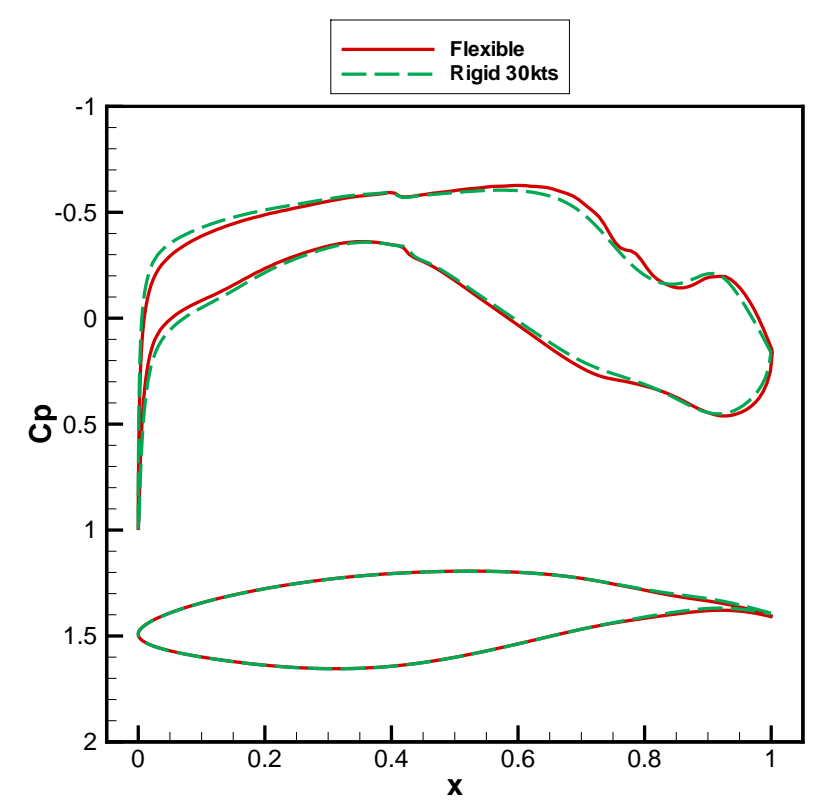

Figure 12: Shapes and pressure coefficient $\mathrm{Cp}$ distributions for the flexible and Rigid 30 kts solutions at 30 kts of boat-speed.

However, as soon as the boat-speed departs from $30 \mathrm{kts}$, the flexible hydrofoil exhibits a lower drag compared to the Rigid $30 \mathrm{kts}$ one. This can be appreciated in Figure 13 which reports the differences in the drag coefficients, with positive values in favor of the flexible case. Note that these consistent improvements were not necessarily expected as the Rigid 30 kts case does not enforce any non-cavitating conditions at boat-speeds other than 30 kts. Therefore, the Rigid 30 kts optimization may have produced a cavitating design with lower drag for other boat-speeds. Note also the higher drag for the flexible hydrofoil, at $20 \mathrm{kts}$ of boat-speed, where the cavitation criterion is 
actually not satisfied by the rigid solution.

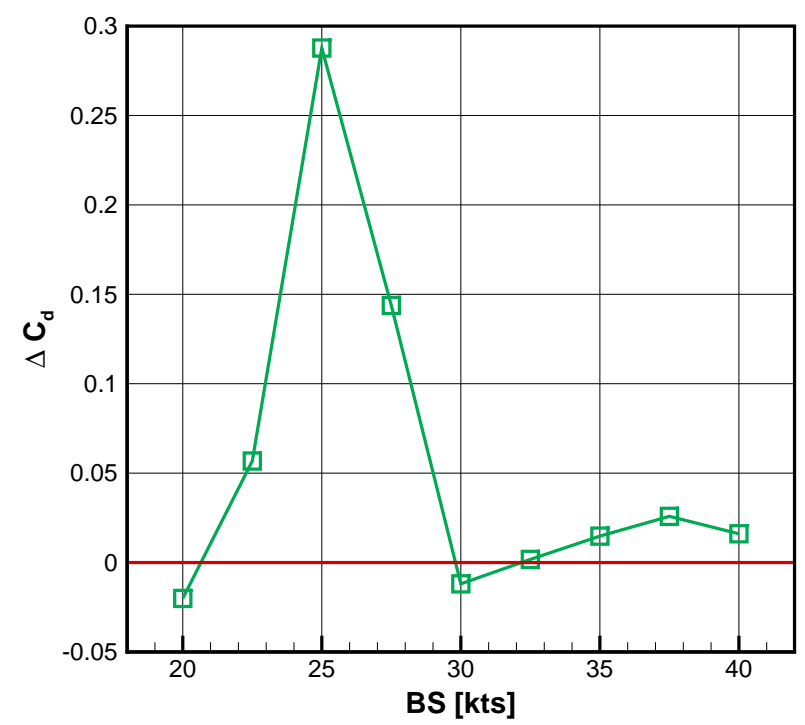

Figure 13: Difference $\Delta \mathrm{C}_{\mathrm{d}}$ in the drags of the Rigid $30 \mathrm{kts}$ and flexible optima, as a function of the boat-speed (BS).

\section{Conclusions}

An advanced multi-design constrained optimization method has been proposed in this paper for the design of a two-dimensional hydrofoil with a flexible trailing edge. Surrogate-model based optimization, with inequality constraints, have been used to enable the optimization of the hydrofoil at a reasonable computational cost. The surrogates are constructed and updated using a limited number of carefully selected resolutions of the fully nonlinear fluid-structure interaction problem. The nonlinear FSI solver used in this work couples a two-dimensional vortex lattice method code for the flow around the hydrofoil with a static structural solver, based on linear stress triangle and Timoshenko beam elements, for the hydrofoil deformations. The optimization involves 4 parameters describing the rotation of a base geometry, and seven elastic parameters describing the properties of the elastic bound between the rigid leading part and trailing edge of the hydrofoil. These parameters are sought to globally minimize the hydrofoil drag forces in selected conditions (boat speeds and lift forces), while constraints were introduced to ensure noncavitating conditions.

The performances of the resulting optimal flexible hydrofoil have been compared to the rigid case optimized for the same set of conditions. It was found that the flexible hydrofoil performs globally better over the whole set of conditions, compared to the rigid one. In fact, the flexible hydrofoil was found to have drag forces lower than that of the rigid one the whole range of boat speeds, except around 20 knots where the two cases have essentially the same drag force. The improvement in the drag force brought by the flexibility is particularly noticeable for the highest boat speeds ( $\geq 35$ knots) and around 25 knots. A closer inspection of the optimal solutions revealed that not only the flexible hydrofoil performs better for most conditions, but its angle of attack varies with the boat speed in a tighter range to achieve the prescribed lift conditions, compared to the rigid case. In particular, the flexible hydrofoil has a much smaller angle of attack at low boat speed, which immediately translates into a higher minimum of pressure and less susceptibility to cavitation.

Overall, the optimization clearly evidences the various advantages of considering a hydrofoil with a flexible trailing edge. In particular, it demonstrates that, if correctly designed, the flexible trailing edge allows for an auto-adaption of the hydrofoil camber line and angle of attack with the boat speed, with a global reduction of the drag and lower cavitation risk.

Future developments will concern the optimization of a threedimensional flexible hydrofoil with the use of more advanced flow models to account for the three-dimensional effects, turbulence, and more complex cavitation criteria. We are currently exploring multi-fidelity strategies [Park et al. 2016] to tempered the increase in the computational costs in the three-dimensional case. Another aspect requiring further investigation is the dependence of the transition point displacement as a function of the hydrofoil angle of attack at lower boat speed range. Indeed, we believe that the flexible trailing edge could be effective in producing enough lift for the platform to take off at a lower boat speed.

\section{Appendix: Preliminary study}

We report in this appendix an optimization result to support the definition of the elastic hydrofoil used in the paper. Specifically, we considered a more general definition of the elastic hydrofoil using six consecutive homogeneous elastic elements in the elastic part ranging in the last $30 \%$ of the hydrofoil chord (see Figure 14). The elastic elements have a fixed size and their respective Young moduli $\mathrm{E}_{\mathrm{i}=1, \ldots, 6}$ are optimized for the same objective function and constraints in (17), together with 3 geometrical parameters (rotations $\mathrm{p}_{1, \ldots, 3}$ defined in Section 3.2), leading to an optimization problem with 9 design variables. The Young moduli are also constrained to remain in a finite (positive) range of available materials.

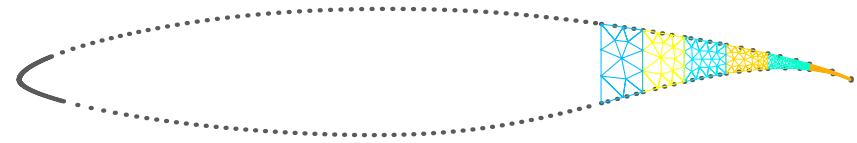

Figure 14: Flexible hydrofoil composed of 6 elastic elements.

The optimal Young moduli $\mathrm{E}_{1, \ldots, 6}$ are reported (as a function of the chord length coordinate), in Figure 15. The values have been divided by a reference value for confidentiality reasons. It is seen that the optimal solution corresponds a localized flexibility, located at $\approx 85 \%$ of the chord length, and with the first and last elastic elements of the flexible hydrofoil having Young moduli equal to the maximum value allowed. In other words, the optimal design presents very stiff upstream part and 
trailing edge, separated by a significantly softer region. An idealized version of this solution is the case of a single homogeneous elastic element described in Section 3.2 and depicted in Figure 2.

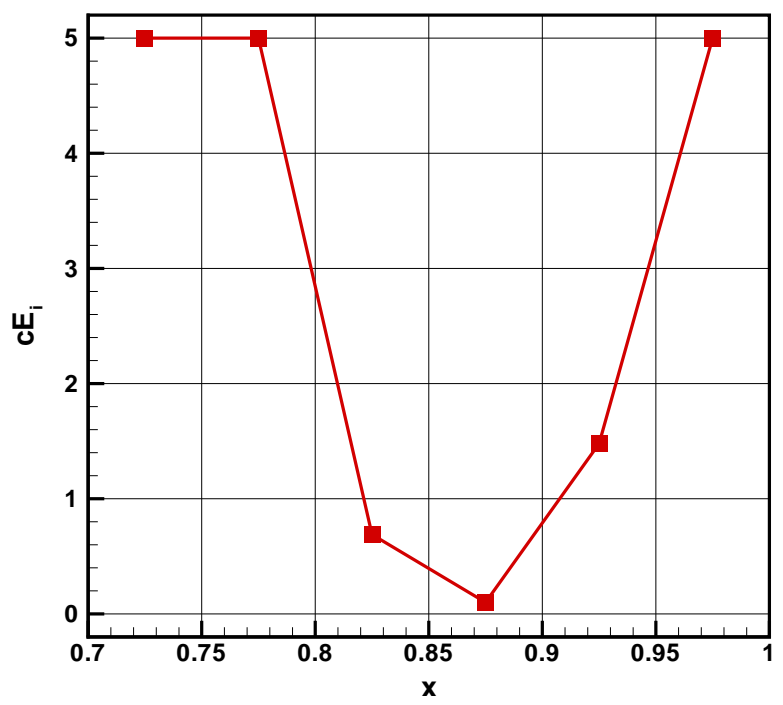

Figure 15: Optimal distribution of the Young modulus (arbitrary units) with the chord length.

\section{References}

P. Richards, A. Johnson, A. Stanton, America's Cup downwind sails-vertical wings or horizontal parachutes?, Journal of Wind Engineering and Industrial Aerodynamics 89 (14-15) (2001) 1565 - 1577, ISSN 0167-6105, doi:Ibibinfo\{doi\}\{http://dx.doi.org/10.1016/S0167-6105(01)00135-0\},

URL http://www.sciencedirect.com/science/article/ pii/S0167610501001350, bluff Body Aerodynamics and Applications.

F. Hueber, G. Caponnetto, C. Poloni, A Passively Morphing Trailing Edge Concept for Sailing Hydrofoil, doi:lbibinfo\{doi\}\{doi:10.20944/ preprints201706.0080.v1\}, preprints, 2017.

A. Ribeiro, A. Awruch, H. Gomes, An airfoil optimization technique for wind turbines, Applied Mathematical Modelling 36 (10) (2012) 4898 - 4907, ISSN 0307-904X, doi:lbibinfo\{doi\}\{http://dx.doi.org/ 10.1016/j.apm.2011.12.026\}, URL http://www.sciencedirect. com/science/article/pii/s0307904X11008146.

D. Srinath, S. Mittal, Optimal aerodynamic design of airfoils in unsteady viscous flows, Computer Methods in Applied Mechanics and Engineering 199 (29-32) (2010) 1976 - 1991, ISSN 0045-7825, doi:Ibibinfo\{doi\}\{http://dx.doi.org/10.1016/j.cma.2010.02.016\}, URL http://www.sciencedirect.com/science/article/pii/ S0045782510000745.

W. Li, L. Huyse, S. Padula, Robust airfoil optimization to achieve drag reduction over a range of Mach numbers, Structural and Multidisciplinary Optimization 24 (1) (2002) 38-50, ISSN 1615-1488, doi:lbibinfo $\{$ doi $\}\{10$. 1007/s00158-002-0212-4\}, URL http://dx.doi.org/10.1007/ s $00158-002-0212-4$.

D. I. Papadimitriou, C. Papadimitriou, Aerodynamic shape optimization for minimum robust drag and lift reliability constraint, Aerospace Science and Technology 55 (2016) 24 - 33, ISSN 1270-9638, doi: Ibibinfo \{doi\}\{http://dx.doi.org/10.1016/j.ast.2016.05.005\}, URL http://www.sciencedirect.com/science/article/pii/ S1270963816301754.

Y. Inukai, K. Horiuchi, T. Kinoshita, H. Kanou, H. Itakura, Development of a single-handed hydrofoil sailing catamaran, Journal of Marine Science and Technology 6 (1) (2001) 31-41, ISSN 1437-8213, doi: lbibinfo\{doi\}\{10.1007/s007730170005\}, URL http://dx.doi.org/ $10.1007 / \mathrm{s} 007730170005$.
M. Kandasamy, D. Peri, S. K. Ooi, P. Carrica, F. Stern, E. F. Campana, P. Osborne, J. Cote, N. Macdonald, N. de Waal, Multi-fidelity optimization of a high-speed foil-assisted semi-planing catamaran for low wake, Journal of Marine Science and Technology 16 (2) (2011) 143-156, ISSN 1437-8213, doi: Ibibinfo \{doi\}\{10.1007/s00773-011-0119-0\}, URL http: //dx.doi.org/10.1007/s00773-011-0119-0.

R. Duvigneau, M. Visonneau, Hydrodynamic design using a derivative-free method, Structural and Multidisciplinary Optimization 28 (2) (2004) 195205, ISSN 1615-1488, doi:lbibinfo \{doi\}\{10.1007/s00158-004-0414-z\}, URL http: / /dx.doi.org/10.1007/s00158-004-0414-z.

M. Sedlar, B. Ji, T. Kratky, T. Rebok, R. Huzlik, Numerical and experimental investigation of three-dimensional cavitating flow around the straight \{NACA2412\} hydrofoil, Ocean Engineering 123 (2016) 357 - 382, ISSN 0029-8018, doi:lbibinfo\{doi\}\{http://dx.doi.org/10.1016/j. oceaneng.2016.07.030\}, URL http://www.sciencedirect.com/ science/article/pii/s0029801816302785.

J.-B. Leroux, O. Coutier-Delgosha, J. A. Astolfi, A joint experimental and numerical study of mechanisms associated to instability of partial cavitation on two-dimensional hydrofoil, Physics of Fluids 17 (5) (2005), doi: \bibinfo\{doi\}\{http://dx.doi.org/10.1063/ 1.1865692\}, URL http://scitation.aip.org/content/aip/ journal/pof2/17/5/10.1063/1.1865692.

O. Coutier-Delgosha, F. Deniset, J. A. Astolfi, J.-B. Leroux, Numerical prediction of cavitating flow on a two-dimensional symmetrical hydrofoil and comparison to experiments, Journal of Fluids Engineering 129 (3) (2007) 279-292.

A. Ducoin, F. Deniset, J. A. Astolfi, J.-F. Sigrist, Numerical and experimental investigation of hydrodynamic characteristics of deformable hydrofoils, Journal of Ship research 53 (4) (2009) 214-226.

D. T. Akcabay, E. J. Chae, Y. L. Young, A. Ducoin, J. A. Astolfi, Cavity induced vibration of flexible hydrofoils, Journal of Fluids and Structures 49 (2014) 463 - 484, ISSN 0889-9746, doi: Ibibinfo\{doi\} \{http://dx.doi.org/10.1016/j.jfluidstructs.2014.05.007\},

URL http://www.sciencedirect.com/science/article/ pii/S0889974614001030.

Q. WEI, H. xun CHEN, R. ZHANG, Numerical research on the performances of slot hydrofoil, Journal of Hydrodynamics, Ser. B 27 (1) (2015) 105 - 111, ISSN 1001-6058, doi:lbibinfo\{doi\}\{http://dx.doi.org/10.1016/ S1001-6058(15)60462-0\}, URL http://www.sciencedirect. com/science/article/pii/S1001605815604620.

M. Drela, XFOIL: An Analysis and Design System for Low Reynolds Number Airfoils, Springer Berlin Heidelberg, Berlin, Heidelberg, ISBN 978-3-64284010-4, 1-12, doi:lbibinfo\{doi\} \{10.1007/978-3-642-84010-4_1\}, URL http://dx.doi.org/10.1007/978-3-642-84010-4_1, 1989.

M. Durand, A. Leroyer, C. Lothodé, F. Hauville, M. Visonneau, R. Floch, L. Guillaume, FSI investigation on stability of downwind sails with an automatic dynamic trimming, Ocean Engineering 90 (2014) 129-139.

T. Simpson, J. Poplinski, N. P. Koch, J. Allen, Metamodels for Computer-based Engineering Design: Survey and recommendations, Engineering with Computers 17 (2) (2001) 129-150, ISSN 1435-5663, doi: Ibibinfo \{doi $\}\{10.1007 /$ PL00007198\}, URL http: / / dx. doi.org/10.1007 / PL0 0007198.

J. P. Kleijnen, Kriging metamodeling in simulation: A review, European Journal of Operational Research 192 (3) (2009) 707 - 716, ISSN 0377-2217, doi:Ibibinfo\{doi\}\{http://dx.doi.org/10.1016/j.ejor.2007.10.013\}, URL http: //www.sciencedirect.com/science/article/pii/ S 0377221707010090

D. R. Jones, M. Schonlau, W. J. Welch, Efficient Global Optimization of Expensive Black-Box Functions, Journal of Global optimization 13 (4) (1998) 455-492.

S. Jeong, M. Murayama, K. Yamamoto, Efficient Optimization Design Method Using Kriging Model, Journal of aircraft 42 (2) (2005) 413-420.

B. Glaz, P. P. Friedmann, L. Liu, Helicopter Vibration Reduction throughout the Entire Flight Envelope Using Surrogate-Based Optimization, Journal of the American Helicopter Society 54 (1) (2009) 12007.

N. Aghajari, M. Schäfer, Efficient shape optimization for fluid-structure interaction problems, Journal of Fluids and Structures 57 (2015) 298- 313.

M. Sacher, F. Hauville, R. Duvigneau, O. L. Maître, N. Aubin, M. Durand, Efficient optimization procedure in non-linear fluid-structure interaction problem: Application to mainsail trimming in upwind conditions, Journal of Fluids and Structures 69 (2017) 209 - 231, ISSN 0889-9746, doi: bibinfo \{doi\} \{http://dx.doi.org/10.1016/j.jfluidstructs.2016.12.006\}, URL 
http://www.sciencedirect.com/science/article/pii/ S0889974616303437.

A. Basudhar, C. Dribusch, S. Lacaze, S. Missoum, Constrained efficient global optimization with support vector machines, Structural and Multidisciplinary Optimization 46 (2) (2012) 201-221, ISSN 16151488, doi:Lbibinfo\{doi\}\{10.1007/s00158-011-0745-5\}, URL http:// dx.doi.org/10.1007/s00158-011-0745-5.

J. Suykens, J. Vandewalle, Least Squares Support Vector Machine Classifiers, Neural Processing Letters 9 (3) (1999) 293-300, ISSN 1573-773X, doi:Lbibinfo\{doi\}\{10.1023/A:1018628609742\}, URL http: / / dx.doi. org/10.1023/A:1018628609742.

C. E. Rasmussen, C. K. I. Williams, Gaussian Processes for Machine Learning, MIT Press, 2006.

N. Hansen, The CMA Evolution Strategy: A Comparing Review, in: Towards a new evolutionary computation, Springer, 75-102, 2006.

D. Huang, T. T. Allen, W. I. Notz, N. Zheng, Global Optimization of Stochastic Black-Box Systems via Sequential Kriging Meta-Models, Journal of global optimization 34 (3) (2006) 441-466.

M. Schonlau, Computer Experiments and Global Optimization, Ph.D. thesis, University of Waterloo, Waterloo, Ont., Canada, Canada, aAINQ22234, 1997.

V. Vapnik, The nature of statistical learning theory, Springer-Verlag New York, Inc., New York, NY, USA, ISBN 0-387-94559-8, 1995.

G. C. Cawley, Leave-one-out cross-validation based model selection criteria for weighted LS-SVMs, in: The 2006 IEEE International Joint Conference on Neural Network Proceedings, IEEE, 1661-1668, 2006.

D. M. Allen, The relationship between variable selection and data augmentation and a method for prediction, Technometrics 16 (1) (1974) 125-127.

G. C. Cawley, N. L. Talbot, Efficient leave-one-out cross-validation of kernel fisher discriminant classifiers, Pattern Recognition 36 (11) (2003) 2585 - 2592, ISSN 0031-3203, doi:lbibinfo\{doi\}\{http://dx.doi.org/10.1016/ S0031-3203(03)00136-5\}, URL http://www.sciencedirect. com/science/article/pii/s0031320303001365.

B. Van Calster, J. Luts, J. A. K. Suykens, G. Condous, T. Bourne, D. Timmerman, S. Van Huffel, Artificial Neural Networks - ICANN 2007: 17th International Conference, Porto, Portugal, September 9-13, 2007, Proceedings, Part II, chap. Comparing Methods for Multi-class Probabilities in Medical Decision Making Using LS-SVMs and Kernel Logistic Regression, Springer Berlin Heidelberg, Berlin, Heidelberg, ISBN 978-3-540-74695-9, 139 148, doi:lbibinfo $\{$ doi $\}\{10.1007 / 978-3-540-74695-9-15\}$, URL http: / / dx.doi.org/10.1007/978-3-540-74695-9-15, 2007.

J. C. Platt, Probabilistic outputs for support vector machines and comparisons to regularized likelihood methods, Advances in large margin classifiers 10 (3) (1999) 61-74.

H.-T. Lin, C.-J. Lin, R. C. Weng, A note on Platt's probabilistic outputs for support vector machines, Machine Learning 68 (3) (2007) 267-276, ISSN 1573-0565, doi: اbibinfo $\{$ doi $\}$ 10.1007/s10994-007-5018-6\}, URL http: //dx.doi.org/10.1007/s10994-007-5018-6.

D. V. Arnold, N. Hansen, A (1+1)-CMA-ES for Constrained Optimisation, in: T. Soule, J. H. Moore (Eds.), GECCO, ACM, ACM Press, Philadelphia, United States, 297-304, doi:lbibinfo\{doi $\}$ \{10.1145/2330163. 2330207\}, URL https: / / hal.inria.fr/hal-00696268, 2012.

T. Bäck, H. P. Schwefel, An Overview of Evolutionary Algorithms for Parameter Optimization, Evolutionary computation 1 (1) (1993) 1-23.

L. Piegl, W. Tiller, The NURBS Book (2Nd Ed.), Springer-Verlag New York, Inc., New York, NY, USA, 1997.

W. Wang, H. Pottmann, Y. Liu, Fitting B-spline Curves to Point Clouds by Curvature-based Squared Distance Minimization, ACM Trans. Graph. 25 (2) (2006) 214-238

J. Van Ingen, The eN method for transition prediction: historical review of work at TU Delft, in: 38th Fluid Dynamics Conference and Exhibit, American Institute of Aeronautics and Astronautics, Seattle, United States, 1-49, 2008.

J. Morgado, R. Vizinho, M. Silvestre, J. Páscoa, \{XFOIL $\}$ vs $\{$ CFD performance predictions for high lift low Reynolds number airfoils, Aerospace Science and Technology 52 (2016) 207 - 214, ISSN 12709638, doi:Ibibinfo\{doi\}\{http://dx.doi.org/10.1016/j.ast.2016.02.031\}, URL http: //www.sciencedirect.com/science/article/pii/ S1270963816300839.

D. MacPhee, A. Beyene, Fluid-structure interaction of a morphing symmetrical wind turbine blade subjected to variable load, International Journal of Energy Research 37 (1) (2013) 69-79, ISSN 1099-114X, doi:Ibibinfo $\{$ doi $\}\{10$. 1002/er.1925\}, URL http://dx.doi.org/10.1002/er.1925.

M. Durand, Interaction fluide-structure souple et légère, application aux voiliers, PhD Thesis, Ecole Centrale de Nantes, 2012.

P. Pedersen, Some properties of linear strain triangles and optimal finite element models, International Journal for Numerical Methods in Engineering 7 (4) (1973) 415-429, ISSN 10970207, doi:lbibinfo\{doi $\}\{10.1002 /$ nme.1620070402\}, URL http: //dx.doi.org/10.1002/nme.1620070402.

F. R. Menter, R. Langtry, S. Likki, Y. Suzen, P. Huang, S. Völker, A correlationbased transition model using local variables-Part I: model formulation, Journal of turbomachinery 128 (3) (2006) 413-422.

C. Park, R. T. Haftka, N. H. Kim, Remarks on multi-fidelity surrogates, Structural and Multidisciplinary Optimization (2016) 1-22ISSN 16151488, doi:lbibinfo\{doi\}\{10.1007/s00158-016-1550-y\}, URL http: / / dx.doi.org/10.1007/s00158-016-1550-y. 